\title{
Manifestes médiologiques
}

Régis Debray, Editions Gallimard, 1994

Pierre Vanier

\section{(2) OpenEdition}

\section{Journals}

Édition électronique

URL : http://journals.openedition.org/edc/2507

DOI : 10.4000/edc.2507

ISSN : 2101-0366

\section{Éditeur}

Université de Lille

Édition imprimée

Date de publication : 1 janvier 1995

Pagination : 195-199

ISSN : 1270-6841

\section{Référence électronique}

Pierre Vanier, «Manifestes médiologiques », Études de communication [En ligne], 16 | 1995, mis en ligne le 22 juin 2011, consulté le 21 septembre 2020. URL : http://journals.openedition.org/edc/2507 ; DOI : https://doi.org/10.4000/edc.2507

Ce document a été généré automatiquement le 21 septembre 2020.

(c) Tous droits réservés 


\title{
Manifestes médiologiques
}

\author{
Régis Debray, Editions Gallimard, 1994
}

\author{
Pierre Vanier
}

\section{Pour une médiologie}

1 Le point de départ de l'ouvrage consiste à poser la question de l'existence et de la fonction des idées et des symboles : pourquoi y a-t-il du symbolique en circulation dans une société ? L'idéologie est ce par quoi toute société s'ouvre sur une valeur suréminente, sa part de symbolique. Deuxième question : comment un symbole abstrait peut-il produire des effets concrets ? La médiologie examine la question de «l'efficacité symbolique», en posant que la médiation détermine la nature du message.

2 L'auteur procède tout d'abord par une série de définitions a contrario, pour se démarquer de la linguistique, de la sémiologie, de la pragmatique et de l'histoire des idées. La médiologie considère l'histoire des idées par le biais des traces matérielles du sens. Elle s'occupe, non des messages, mais de leur cheminement, diffusion, propagation. C'est donc une pragmatique de la pensée - du dire au faire -, qui répond à la question : «Comment une idée sur le monde peut-elle transformer un état du monde ?» L'étude des médiations consiste à mettre en rapport les productions symboliques d'une société et la physique des traces (saisie, archivage, circulation).

3 La médiologie est l'étude des médiations, soit l'ensemble dynamique des procédures et corps intermédiaires qui s'interposent entre une production de signes et une production d'événements. On comprend qu'elle est d'emblée transdisciplinaire, et que la question de l'opérativité des signes est totalisante : elle réunit les trois aspects technique, sémantique et politique. Par ailleurs, elle récuse le clivage qui sépare l'outil technique et la vie de la culture, et qui entretient ce vieux dualisme qui sépare l'univers des objets et l'univers des sujets.

Dans la techno-histoire de la culture, le médiologue repère, par invariants et variations, des cycles de périodisation, appelés médiasphères, soit trois grandes périodes de l'efficacité symbolique : la logosphère (écrit), la graphosphère (imprimé), la vidéosphère (audiovisuel). 
5 La notion de médiasphère (milieu techno-culturel) - à la fois dispositif logistique et disposition psychologique - échappe aussi bien au dualisme esprit/matière, qu'à l'essentialisme technocentriste. Certes, le support prime sur le symbole, mais la technique n'est pas une valeur immanente. Le progrès technique est de l'ordre du fait, il est étranger à l'ordre de la valeur. Ni structure (immanence), ni système (autorégulation), ni champ (Bourdieu), la notion de média-sphère est synthétique, elle englobe des déterminations à caractère à la fois social, économique, technique et politique ; elle est assimilable à une biosphère de l'esprit.

\section{Un nouveau tournant?}

6 Régis Debray formule de vives critiques à l'encontre de deux disciplines concurrentes : la théorie de la communication, et la sémiologie. La théorie de l'information est un modèle technocentriste. Il n'est pas extrapolable au-delà de l'univers mécanique vers la sphère des croyances, des convictions. L'information n'a d'existence que rapportée à des champs sociaux. Si la sémiologie a permis d'établir l'autonomie du langage (et par extension de toute organisation signifiante) comme système de relations internes, dissipant ainsi l'illusion selon laquelle les systèmes de signes ne sont qu'un double des réalités auxquelles ils se réfèrent, la médiologie doit chercher aujourd'hui à retrouver dans les signes une référence forte au monde, et à mettre au jour les socles matériels des cultures (traces, parcours, instrumentation, voies de communication). Au postulat structuraliste selon lequel toute réalité doit s'analyser comme un ensemble de relations entre des éléments (rejoignant ainsi le modèle cybernéticien de la communication), au postulat immanentiste qui conduit à l'expulsion du référent, Régis Debray reproche de clôturer la langue sur elle-même. Pour le médiologue, c'est la pragmatique des usages qui détermine la sémiotique des codes. Les démarches formalistes de la sémiologie ont méconnu la nécessaire « déférence envers les objets et les techniques».

7 Le tournant sémiotique a marqué le passage du quoi au comment. La médiologie se tourne maintenant vers les conditions matérielles du comment. Contre le dualisme de la sémiologie (choses/esprit), la médiologie pose que les agencements matériels du message sont constitutifs du message lui-même, elle entreprend de remonter de la signifiance à l'usinage.

\section{Un parcours philosophique}

8 La Raison a toujours été conçue comme la propagation naturelle d'une Lumière. De la révélation des Ecritures à Voltaire dissipant les ténèbres, et jusqu'à aujourd'hui, la vérité se conçoit comme une lumière immanente à elle-même, le vrai comme transcendant aux opérations de sa découverte. L'immédiateté solaire du vrai voit dans la lumière l'évidente métaphore de la Raison, ce qui rend celle-ci aveugle à ses propres instruments.

9 C'est là que prend sa source l'illusion idéaliste selon laquelle les idées dirigent le monde, ce qui revient à prendre l'effet pour la cause. Or, c'est la méthode de divulgation qui fait la doctrine. Le dire peut-il être la cause responsable du faire ? Non. La pertinence d'une thèse ne lui est pas immanente. Elle est co-produite par sa réception, par sa relative adéquation avec les attentes sociales de son milieu. Il faut 
donc une écologie de la sémiosphère, à quoi prétend la médiologie - interdiscipline de synthèse - qui travaille à une vue d'ensemble des rapports supports/symboles.

La médioéthique qui se propose ainsi doit d'abord se guérir du prurit technophobe. Contre la tradition humaniste du dualisme (forme/matière, esprit/corps, individu/ société), le médiologue pose que la technique et la culture ne sont pas extérieures l'une à l'autre. Pas plus qu'il n'y a d'autonomie du fait technique, il n'y a d'autonomie du fait culturel. L'homme fabrique l'outil qui fabrique l'homme. Il faut réhabiliter la technique et penser la culture à partir de la technique. L'homo sapiens est un homo faber (LeroiGourhan). L'extériorisation de l'homme en machines et en technologies n'est pas une dénaturation déshumanisante. Elle constitue précisément l'évolution ethnique de l'humanité. Le faber fait le sapiens, l'extérieur l'intérieur. La nature de l'homme, c'est de n'avoir pas de nature, et d'inverser, par la genèse technique, cette déficience d'être en avantage spécifique.

11 Reconnaître que les contenus de connaissance ne sont pas indifférents aux «technologies de l'intelligence» qu'ils induisent fait subir une blessure narcissique à une certaine réflexion humaniste sur le médium comme simple moyen. Une conception instrumentale de l'instrument permet de croire que le sujet règne sur son milieu, comme capitaine en son navire. Or c'est mon milieu technique de production et de transmission (médiasphère) qui est le moteur de mes idées. Que reste-t-il alors de la souveraineté de l'esprit, s'il ne vaut que par les médiations qu'il engendre et à travers lesquelles il passe ? Les objets techno-culturels ont bien, en effet, leur logique propre. Nous n'avons pas prise sur le devenir technoscientifique, lequel a, en retour, prise sur nous : dissymétrie vexatoire pour l'orgueil du sujet monarchique. Se pose alors la question du libre arbitre. Comment éviter que l'esprit ne devienne l'outil de ses outils? Il faut reconnaître aux machines plus, pour leur concéder moins. La primauté d'une volonté morale et politique passe par la prise en compte des propriétés objectives des appareillages et de leur économie propre. La connaissance médiologique met en évidence notre capacité à modifier le milieu qui nous modifie. La médiologie est un métier de pointe.

\section{DEFENDRE L'IMAGE}

La médiologie - remontée vers les régimes de croyance des pratiques figuratives - est une histoire des efficacités visuelles sur le double plan de l'outillage et des croyances. Elle consiste à multiplier les ponts entre les champs esthétique et technique. Une pragmatique de l'image, en posant la question de l'efficacité iconique - et non celle de l'essence, ni celle du sens - fait apparaitre trois grands cycles dans le pouvoir symbolique de l'image : pratiques dévotionnelles (l'idole), pratiques artistiques (l'œuvre), pratiques informationnelles (la pub).

Théorie des effets, la médiologie définit la nature des images par leurs procédés matériels de fabrication et de diffusion. Contre la sémiologie, la médiologie affirme que «image n'est pas langage». La généralisation de l'ordre du langage au domaine de la figuration iconique est abusive. On peut décrypter une image, non la réduire à un code. La plus-value figurative est dans le déficit de code. Une dualité matricielle oppose les deux ordres de la représentation (les images et les mots). Il faut mettre à jour le propre des figures face aux discours. Historiquement, l'image sert d'abord à conjurer la mort. La sacralité initiale de l'image tient à son ambivalence magique : faire survivre une âme 
spirituelle dans un support matériel. L'image introduit le monde invisible dans le monde visible. De la résurrection par l'image (l'idole), il reste l'image comme résurrection (la photo).

14 L'élucidation des origines théologiques de la représentation est tout aussi nécessaire que celle de ses origines anthropologiques. Contre le tabou iconophobe du monothéisme, le christianisme érige en dogme de l'Incarnation l'accès au Verbe par l'image, corollaire de l'accès à Dieu par le Christ. Si pas d'incarnation, pas de représentation dans l'occident chrétien. Hollywood est né à Bizance. Le décollement d'avec l'ordre théologique assure la transition de l'idole à l'œuvre. Naissance de l'art et de l'artiste.

\section{AUTEUR}

\section{PIERRE VANIER}

Pierre Vanier, DEA Sciences de la communication - Lille III 